\title{
CONTRAST ENHANCEMENT USING PHASE TRANSITION INFORMATION AND TOTAL VARIATION
}

\author{
Serdar Cakir, A.Enis Cetin* \\ Department of Electrical and Electronics Engineering \\ Bilkent University, Ankara, 06800, TURKEY \\ E-mail: $\{$ cakir,cetin\}ee.bilkent.edu.tr
}

\begin{abstract}
Contrast enhancement is an important preprocessing step for the analysis of images. The main aim of contrast enhancement techniques is to increase the visibility of the objects by modifying the spatial characteristics of the image. In this paper, phase transition based contrast enhancement framework is proposed to overcome the limitations of existing image enhancement techniques. The proposed image enhancement framework transforms the changes in image phase into the variations of magnitude to enhance the structural details of the image and to improve visibility. In addition, the concept of Selective Variation (SV) technique is introduced and enhancement parameters are optimized using SV. The experimental studies that were carried out on TID2008 dataset, show that the proposed scheme obtains promising results on contrast enhancement.
\end{abstract}

Index Terms - Contrast Enhancement, Image Phase Information, Fourier Transform.

\section{INTRODUCTION}

Contrast enhancement has been widely used in various image processing, and computer vision applications [1]. The main aim of this process is to increase the visibility of the objects in order to assist the manual or automated tasks including object analysis, detection, segmentation, and recognition. Image histogram based approaches have been widely utilized for contrast enhancement [1]. Early global Histogram Equalization (HE) techniques modify the histogram directly to perform contrast enhancement. However, global HE approaches may introduce enhancement artifacts in the results while failing to enhance the local details. In order to eliminate the enhancement artifacts, the researchers have tried to modify the histogram in an adaptive manner $[2,3]$. In the adaptive HE methods [3] the image is divided into sub-blocks and $\mathrm{HE}$ is carried out within these sub-blocks. The adaptive HE methods may result in a "checker-board" effect due to

*A. Enis Cetin is on leave from Bilkent University. He is with the Department of Electrical \& Computer Engineering, University of Illinois at Chicago, Chicago, IL 60607, USA. local contrast changes when combining the sub-blocks. The use of overlapping blocks may eliminate the grid-shaped artifacts at the expense of increasing computational complexity and memory. Contrast limited adaptive HE (CLAHE) [2,3] was proposed to overcome the limitations of HE approaches. The CLAHE method balances the contrast and eliminates artifacts caused by inappropriate mapping of grayscale values. In general, the CLAHE method is preferred in offline applications due to its computational load. Therefore, there have been several attempts which try to realize the algorithm efficiently for real-time applications [4,5].

In this work, an image enhancement framework is proposed to enhance the visual details in images. In the proposed image enhancement framework, the Discrete Fourier Transform (DFT) of the image is computed to determine the image phase information. Locations of high phase transitions are important for images [6]. Such frequency locations are extracted in DFT domain. To transform the phase differences into space domain amplitude variations, the frequency components where the phase transitions are significant are amplified with large weights. After the calculation of the inverse DFT, the enhanced version of the image is obtained. The parameters of the algorithm are determined by the Total Variation (TV) [7] based approach. In this study, the concept of Selective Variation (SV) is introduced. The SV method minimizes the variations in local regions of interest to achieve significant transitions around edges.

\section{CONTRAST ENHANCEMENT BASED ON FOURIER PHASE TRANSITION INFORMATION}

In this study, a contrast enhancement technique which utilizes the image phase information is proposed. The method aims to increase the weight of the frequencies according to their phase transitions. Highly structured textures and sharp transitions in the image domain contribute to the high frequency components of an image. To emphasize high frequency components containing important hidden details, Fourier domain modifications can be carried out while preserving the conjugatesymmetric nature of the Fourier transform coefficients. 
In the proposed algorithm, the high frequency components are emphasized by making use of the variations in image phase. Let $x[m, n] \in \mathbb{R}^{M \times N}$ be the $M \times N$ dimensional image. The algorithm starts by calculating the two dimensional $\hat{M} \times \hat{N}$-point Discrete Fourier Transform (2D-DFT) $X[u, v]$ of the image $x[m, n]$. In order to take advantage of the Fast Fourier Transform (FFT) algorithm, the DFT parameters should be selected as $\hat{M}=2^{p} \geqslant M, \hat{N}=2^{q} \geqslant N$, where $p$ and $q$ are positive integers. Let $X[u, v]$ be:

$$
\begin{aligned}
& X[u, v]=|X[u, v]| e^{j \phi[u, v]} \\
& u=0,1, \ldots, \hat{M}-1 \& v=0,1, \ldots, \hat{N}-1
\end{aligned}
$$

Note that, the 2D-DFT matrix is shifted to have the low frequency components at the center. Since low frequency components correspond to the smooth and constant regions of an image, manipulations on low frequency components may degrade the quality of the image by causing undesired artifacts on smooth regions. Therefore, the weighting scheme only modifies the high frequency components. To determine the high frequency components to be amplified, a high-pass mask $H[u, v]$ with cut-off frequency indices $u_{c}$ and $v_{c}$ is used:

$$
H[u, v]=\left\{\begin{array}{cc}
0, & \frac{\hat{M}}{2}-u_{c} \leq u \leq \frac{\hat{M}}{2}+u_{c} \\
1, & \frac{\hat{N}}{2}-v_{c} \leq v \leq \frac{\hat{N}}{2}+v_{c} \\
1, & \text { otherwise }
\end{array}\right.
$$

The high frequency components are emphasized by a weighting scheme which is obtained by calculating the transitions of image phase information between neighboring frequency components. The phase transitions correspond to the phase gradient along both horizontal and vertical axes. The phase gradient $\partial_{\phi}[u, v]$ is calculated as follows:

$$
\begin{gathered}
\partial_{\phi}[u, v]=|\phi[u, v]-\phi[u-1, v]| \\
+|\phi[u, v]-\phi[u, v-1]|
\end{gathered}
$$

After calculating the phase gradient, neighboring frequency components which provide significant phase transitions are determined. Let $S$ be the set of spatial locations in the frequency domain at which the corresponding phase transitions exceed the threshold $\tau_{\phi}$ :

$$
S=\left\{(u, v) \mid \partial_{\phi}[u, v]>\tau_{\phi}\right\}
$$

By using the set $S$, the phase transition based weight matrix $W_{p}(u, v)$ is determined as follows:

$$
W_{p}[u, v]=\left\{\begin{array}{cc}
\alpha \partial_{\phi}[u, v], & \text { for }(u, v) \in S \\
1, & \text { otherwise }
\end{array}\right.
$$

where $\alpha$ is a scaling factor. Since direct thresholding is utilized in Equation 4, the weight matrix $W_{p}[u, v]$ may have sharp transitions which may cause undesired artifacts in the reconstruction. In order to smooth the discontinuities in $W_{p}[u, v]$, a 2D-Gaussian function $(g[u, v])$ with parameters $\mu$ and $\sigma$ is utilized as follows:

$$
W_{f}[u, v]=W_{p}[u, v] * g[u, v]
$$

where " $*$ " is the convolution operator. Afterwards, the final weight matrix $W$ is selectively obtained by making use of $H[u, v]$ as follows:

$$
W[u, v]=\left\{\begin{array}{cc}
W_{f}[u, v], & \text { if } H[u, v]=1 \\
1, & \text { otherwise }
\end{array}\right.
$$

Then, the weight matrix $W[u, v]$ is applied to the spectrum matrix $X[u, v]$ to obtain $X_{C}[u, v]=W[u, v] X[u, v]$. In this manner, the image spectrum is modified in terms of magnitude by using the phase transitions in neighboring frequency components. The inverse DFT (IDFT) of $X_{C}[u, v]$ is calculated to obtain the contrast-enhanced image $x_{C}[m, n]$. Here, the IDFT is realized using the inverse FFT (IFFT) algorithm.

The enhancement level of the image $x_{C}[m, n]$ is determined by the following parameters; the phase filter cut-off frequency indices $u_{c}, v_{c}$, scaling coefficient $\alpha$, phase shift $\theta$, phase gradient threshold $\tau_{\phi}$, and parameters of the Gaussian smoothing function $\mu, \sigma$. Therefore, it is important to select the appropriate parameter set which maximizes the image contrast. In the algorithm, since we amplify the high frequency components to reveal the important details, the noise present in these components is also increased. Therefore, a TV based minimization process can be a useful solution. In most image processing applications, the TV of the original image $x[m, n]$ is minimized [6-9]. There have been also several attempts to utilize weighted variation based frameworks to obtain better results $[10,11]$. In our study, instead of minimizing the TV over the entire image, we select "busy" regions of the image and carry out the optimization process over selected regions. Therefore, background pixels are excluded in the optimization process. Since only partial image information is used in the calculation of "variation", we define "Selective Variation" (SV) as follows:

$$
\begin{aligned}
S V\left(x_{C}\right)=S & {[m, n] \cdot\left(\sum_{m, n}\left|x_{C}[m+1, n]-x_{C}[m, n]\right|\right.} \\
& \left.+\sum_{m, n}\left|x_{C}[m, n+1]-x_{C}[m, n]\right|\right)
\end{aligned}
$$

where the selected pixels are obtained as follows:

$S[m, n]=\left\{\begin{array}{cc}1, & x[m, n]>\frac{1}{b^{2}} \sum_{i=m-\frac{b}{2},}^{m+\frac{b}{2}} \sum_{j=n-\frac{b}{2},}^{n+\frac{b}{2}} x[i, j]+\beta \\ 1, & x[m, n]<\frac{1}{b^{2}} \sum_{\substack{i=m-\frac{b}{2}, \\ \text { otherwise }}}^{m+\frac{b}{2}} \sum_{j=n-\frac{b}{2},}^{n+\frac{b}{2}} x[i, j]-\beta \\ 0, & \end{array}\right.$ 
where $b$ denotes the overlapping local window size and $\beta$ is an additive constant which determines a deviation limit for each pixel from the average values of their local blocks.

The ideal parameter set for the proposed enhancement algorithm is obtained by solving the following minimization problem:

$$
\begin{gathered}
x_{C}^{*}[m, n]=\underset{u_{c}, v_{c}, \theta, \tau_{\phi}, \alpha}{\arg \max } S V\left(x_{C}[m, n]\right) \\
\text { subject to } u_{c}<\hat{M}, v_{c}<\hat{N} \\
\frac{\pi}{K_{1}} \leqslant \theta \leqslant \frac{\pi}{K_{2}}, \\
c_{1} \pi \leqslant \tau_{\phi} \leqslant c_{2} \pi, \text { and } \\
a_{1} \leqslant \alpha \leqslant a_{2}
\end{gathered}
$$

where $x_{C}^{*}[m, n]$ is the enhanced image obtained using the ideal parameter set. $K_{1}$ and $K_{2}$ determine the phase interval added to the high frequency components of the spectrum. The $c_{1}, c_{2}$ coefficients determine the interval for significant phase transitions, and $a_{1}, a_{2}$ values are used to limit the scaling coefficient $\alpha$ used in the weighting stage.

\section{EXPERIMENTAL STUDIES}

The ideal parameter set which minimizes Equation 10 is determined by covering the following parameter space using a grid-search framework:

$$
\begin{aligned}
& u_{c} \in[1, \hat{M}], v_{c} \in[1, \hat{N}], \theta \in\left(\frac{\pi}{2^{10}}, \frac{\pi}{2^{3}}\right), \\
& \alpha \in(1,3), \tau_{\phi} \in(0.5 \pi, 2.5 \pi)
\end{aligned}
$$

The proposed framework is implemented in MATLAB environment on a computer containing Intel(R) Core(TM) i5 3.40 $\mathrm{GHz}$ processor, $8 \mathrm{~GB}$ RAM with Microsoft Windows 7 operating system. The enhancement of a single image of size $512 \times 512$ takes approximately 0.1351 seconds. Experimental results show that, the proposed algorithm obtains satisfactory enhancement results, if the parameters are selected as $u_{c}=v_{c}=2, \theta=\pi / 32, \alpha=2, \tau_{\phi}=\pi / 4$.

In order to test the effectiveness of the proposed contrast enhancement framework, a comparative performance analysis is carried out by comparing the performance of the proposed technique with the performances of baseline enhancement methodologies. In the performance analysis, 25 reference images of size $384 \times 512$ of the TID2008 image dataset [12] are used after performing a contrast reduction process.

The proposed phase-based enhancement framework is compared with several CLAHE techniques which model the results with different distributions (Rayleigh, uniform, exponential). In addition, a balanced CLAHE approach (BCLAHE) [13] is employed in the comparison. Frequency domain approaches based on homomorphic filtering [14] and Aare Mällo method (MEAM) [15] are also utilized in the tests. The last enhancement method used is the comparison is the Retinex based-enhancement framework [16]. The enhancement outputs of the proposed and baseline algorithms on a sample image are presented in Figure 1.

By looking at the enhancement results presented in Figure 1 one can observe that, BCLAHE, CLAHE exponential, and the proposed phase-based enhancement algorithms provide noticeable improvements on image contrast. However, it can be easily observed that, the proposed scheme enhances the foreground/background separability better than the baseline techniques.

In order to obtain a fair and quantitative performance analysis, "Color Enhancement Factor (CEF)" [18], Histogram Flatness (HF) \& Histogram Spread (HS) [19], and Measure of Enhancement (EME) [20] objective measures are used in the performance comparisons. These objective measures are selected to quantify the colorfulness, to investigate the intensity distribution and to measure the level of local enhancement in the enhanced image. These measures provide quantitative evaluation of the enhancement level by utilizing a "global" approach where the enhancement quality is evaluated by a single score for the whole image. In addition to the global approach, a more comprehensive performance evaluation methodology is proposed. In this approach, the input image is divided into $N \times N$ non-overlapping sub-blocks. Then, the objective performance measures are calculated for each sub-block. Using the performance scores obtained for each sub-block, the "performance rate plot" of each method is evaluated. The performance rate plots compute the percentage of sub-blocks which provide objective scores higher than the prescribed threshold values. The performance rate plots are similar to the success rate plots used in visual object tracking applications [21].

In the objective evaluations, the performance of the proposed scheme is compared with the performances of the baseline techniques using the objective performance measures computed over 25 images. The average performance of each enhancement framework corresponding to each objective measure is presented in Table 1 .

Table 1. Average performance of the enhancement techniques on the objective performance measures.

\begin{tabular}{r|cccc}
\hline & \multicolumn{4}{|c}{ Objective Performance Measures } \\
& CEF & HF & HS & EME \\
\hline BCLAHE [13] & $\mathbf{5 . 4 2 7 6}$ & $\mathbf{0 . 2 7 7 6}$ & 0.1749 & $\mathbf{1 5 . 4 6 0 3}$ \\
CLAHE Rayleigh [3] & 1.5329 & 0.0189 & 0.0595 & 6.0973 \\
CLAHE Uniform [3] & 2.2905 & 0.0502 & 0.0918 & 8.6524 \\
CLAHE Exponential [3] & 2.2877 & 0.0498 & 0.0907 & 9.4899 \\
Homomorphic Filtering [17] & 2.7733 & 0.0689 & 0.0463 & 10.1069 \\
MEAM [15] & 4.9145 & 0.1483 & 0.1322 & 10.6634 \\
Retinex [16] & $\mathbf{6 . 6 6 2 3}$ & 0.1997 & $\mathbf{0 . 1 7 9 4}$ & 8.8720 \\
Proposed & 4.3041 & $\mathbf{0 . 4 4 2 7}$ & $\mathbf{0 . 1 7 6 2}$ & $\mathbf{1 8 . 5 0 9 4}$ \\
\hline
\end{tabular}

In Table 1, the highest two scores obtained for each objective performance measure are written in bold font. By looking at the results presented in Table 1, one can conclude 


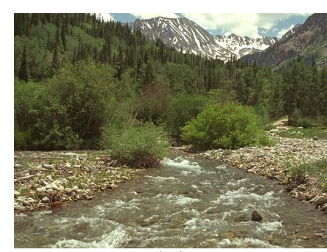

(a)

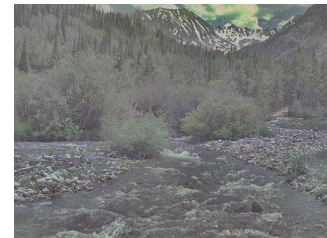

(f)

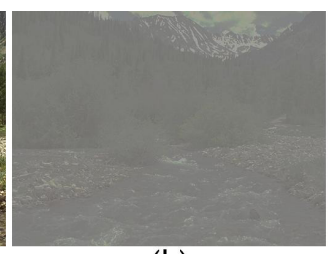

(b)

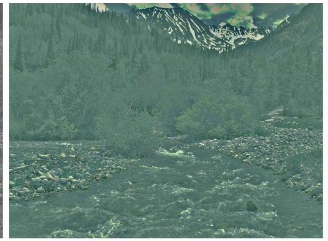

(g)

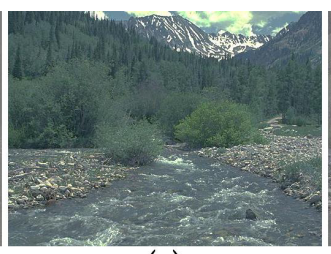

(c)

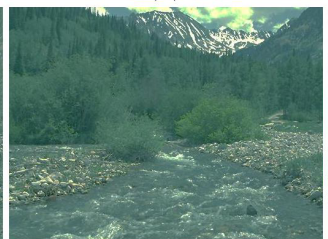

(h)

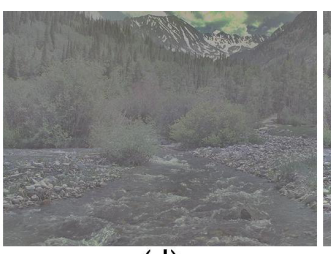

(d)

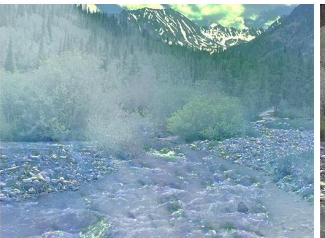

(i)

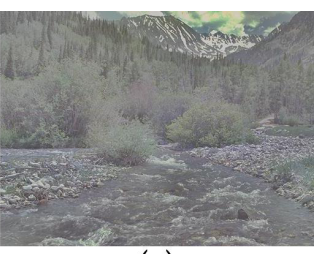

(e)

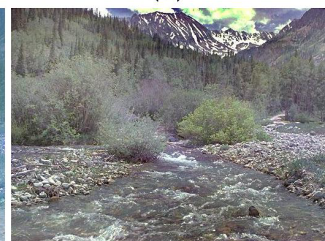

(j)

Fig. 1. The enhancement results of a sample image of TID2008 image dataset by the proposed and baseline enhancement algorithms. (a) Original Image, (b) Low Contrast Image (c) BCLAHE [13], (d) CLAHE Rayleigh [3], (e) CLAHE Uniform [3], (f) CLAHE Exponential [3], (g) Homomorphic Filtering [17], (h) MEAM [15], (i) Retinex [16], (j) Proposed.

that the proposed technique outperforms the baseline methods in most of the objective quality measures. The BCLAHE and CLAHE exponential frameworks also provide satisfactory performance on several performance measures. Although the results presented in Table 1, which are obtained by the global approach, give an idea about the enhancement performance, a more comprehensive evaluation approach is utilized by constructing performance rate plots for each objective measure. The performance rate plots corresponding to each objective performance measure and enhancement algorithm are presented in Figure 2.

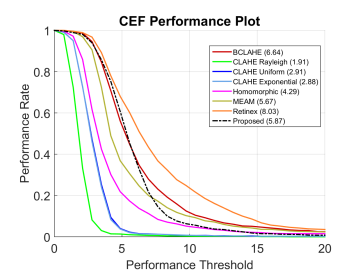

(a)

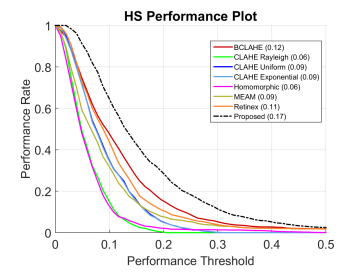

(c)

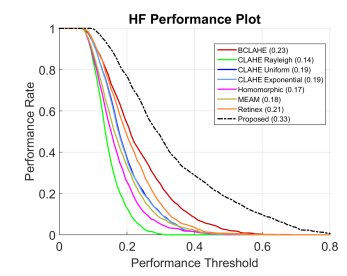

(b)

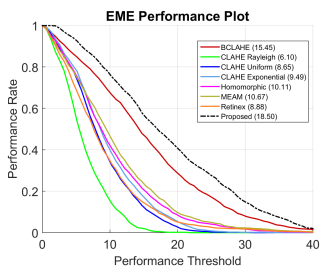

(d)
Fig. 2. Performance rate plots $(N=32)$ for objective performance measures. Area Under Curve (AUC) measures for each enhancement framework are presented in the legend of the figure. Higher values of AUC measure correspond to higher performance.
The performance rate plots for the CEF measure (Figure 2(a)) show that the Retinex and BCLAHE enhancement frameworks outperform the remaining techniques on color enhancement. Although the Retinex technique provides better performance on color enhancement, it introduces color artifacts on the images. By looking at the histogram based performance plots (HF\&HS) presented in Figure 2(b) and Figure 2(c), one can conclude that the proposed phase-based scheme outperforms the baseline techniques on histogram based performance measures. Although CLAHE based enhancement methods operate directly on the image histogram, the proposed algorithm provides better histogram flatness and histogram spread performances without using any histogram information. The results show the efficiency of the proposed algorithm by intrinsically balancing the histogram for better enhancement performance. The EME performance plots presented in Figure 2(d) show that the proposed enhancement framework achieves the highest performance on local contrast-based EME measure. The BCLAHE technique also provides an acceptable performance on the EME measure.

\section{CONCLUSION}

In this article, an image enhancement framework which makes use of the image phase information is proposed to improve the structural details in images. In the proposed scheme, phase variations are translated into amplitude changes in order to reveal the suppressed details present in the high frequency components. In this way, highly structured regions of the image become more significant than the background. The performance of the proposed technique is evaluated on the TID2008 image dataset. Experimental results show that the proposed scheme obtains satisfactory performance on the objective performance evaluations. 


\section{REFERENCES}

[1] Anil K. Jain, Fundamentals of Digital Image Processing, Prentice-Hall, Englewood Cliffs, 1989.

[2] Stephen M. Pizer, E. Philip Amburn, John D. Austin, Robert Cromartie, Ari Geselowitz, Trey Greer, Bart Ter Haar Romeny, and John B. Zimmerman, "Adaptive histogram equalization and its variations," Comput. Vision Graph. Image Process., vol. 39, no. 3, pp. 355-368, Sept. 1987.

[3] Karel Zuiderveld, "Graphics gems iv," chapter Contrast Limited Adaptive Histogram Equalization, pp. 474-485. Academic Press Professional, Inc., San Diego, CA, USA, 1994.

[4] Ali M. Reza, "Realization of the Contrast Limited Adaptive Histogram Equalization (CLAHE) for RealTime Image Enhancement," Journal of VLSI signal processing systems for signal, image and video technology, vol. 38, no. 1, pp. 35-44, 2004.

[5] S. M. Pizer, R. E. Johnston, J. P. Ericksen, B. C. Yankaskas, and K. E. Muller, "Contrast-limited adaptive histogram equalization: speed and effectiveness," in [1990] Proceedings of the First Conference on Visualization in Biomedical Computing, May 1990, pp. 337345.

[6] M. Tofighi, O. Yorulmaz, K. Kse, D. C. Yldrm, R. etin Atalay, and A. E. etin, "Phase and tv based convex sets for blind deconvolution of microscopic images," IEEE Journal of Selected Topics in Signal Processing, vol. 10, no. 1, pp. 81-91, Feb 2016.

[7] Leonid I. Rudin, Stanley Osher, and Emad Fatemi, "Nonlinear total variation based noise removal algorithms," Physica D: Nonlinear Phenomena, vol. 60, no. 1, pp. $259-268,1992$.

[8] Antonin Chambolle, "An algorithm for total variation minimization and applications," Journal of Mathematical Imaging and Vision, vol. 20, no. 1, pp. 89-97, 2004.

[9] K. Kose, V. Cevher, and A. E. Cetin, "Filtered variation method for denoising and sparse signal processing," in 2012 IEEE International Conference on Acoustics, Speech and Signal Processing (ICASSP), March 2012, pp. 3329-3332.

[10] Yan Liu, Jianhua Ma, Yi Fan, and Zhengrong Liang, "Adaptive-weighted total variation minimization for sparse data toward low-dose x-ray computed tomography image reconstruction," Physics in Medicine \& Biology, vol. 57, no. 23, pp. 7923, 2012.
[11] Xueyang Fu, Delu Zeng, Yue Huang, Xiao-Ping Zhang, and Xinghao Ding, "A weighted variational model for simultaneous reflectance and illumination estimation," in Proceedings of the IEEE Conference on Computer Vision and Pattern Recognition, 2016, pp. 2782-2790.

[12] N. Ponomarenko, V. Lukin, A. Zelensky, K. Egiazarian, M. Carli, and F. Battisti, "TID2008 - A Database for Evaluation of Full-Reference Visual Quality Assessment Metrics," Advances of Modern Radioelectronics, vol. 10, pp. 30-45, 2009.

[13] Francesco Branchitta, Marco Diani, Giovanni Corsini, and Antonio Porta, "Dynamic-range compression and contrast enhancement in infrared imaging systems," $\mathrm{OP}$ tical Engineering, vol. 47, no. 7, pp. 076401-076401, 2008.

[14] Tarun Kumar Agarwal, Mayank Tiwari, and Subir Singh Lamba, "Modified histogram based contrast enhancement using homomorphic filtering for medical images," in Advance Computing Conference (IACC), 2014 IEEE International. IEEE, 2014, pp. 964-968.

[15] Carl Salvaggio Seth Weith-Glushko, "Quantitative analysis of infrared contrast enhancement algorithms," 2007.

[16] Zia-ur Rahman, Daniel J Jobson, and Glenn A Woodell, "Retinex processing for automatic image enhancement," Journal of Electronic Imaging, vol. 13, no. 1, pp. 100110, 2004.

[17] Igor Belykh, Homomorphic Filtering for Radiographic Image Contrast Enhancement and Artifacts Elimination, pp. 423-430, Springer International Publishing, 2015.

[18] Yan-Yu Fu, Color image quality measures and retrieval, New Jersey Institute of Technology, 2006.

[19] A. K. Tripathi, S. Mukhopadhyay, and A. K. Dhara, "Performance metrics for image contrast," in 2011 International Conference on Image Information Processing, Nov 2011, pp. 1-4.

[20] Suneet Gupta and Rabins Porwal, "Appropriate contrast enhancement measures for brain and breast cancer images," International journal of biomedical imaging, vol. 2016, 2016.

[21] Yi Wu, Jongwoo Lim, and Ming-Hsuan Yang, "Online object tracking: A benchmark," in Proceedings of the IEEE conference on computer vision and pattern recognition, 2013, pp. 2411-2418. 